\title{
Safety and utility of magnetic resonance imaging in patients with cardiac implantable electronic devices
}

\author{
Jordan B. Strom, MD*, ${ }^{\star}$, Jill B. Whelan, $\mathbf{M D}^{\dagger}, \ddagger$, Changyu Shen, $\mathrm{PhD}^{\star}, \dagger$, Shuang Qi Zheng, \\ $\mathrm{MS}^{\dagger}$, Koenraad J. Mortele, MD§, and Daniel B. Kramer, MD, MPH ${ }^{\star} \dagger$ \\ ${ }^{*}$ Richard A. and Susan F. Smith Center for Cardiovascular Outcomes Research, Division of \\ Cardiovascular Medicine, Beth Israel Deaconess Medical Center, Boston, Massachusetts \\ tHarvard Medical School, Boston, Massachusetts \\ ‡Division of Cardiovascular Medicine, Beth Israel Deaconess Medical Center, Boston, \\ Massachusetts \\ §Department of Radiology, Divisions of Abdominal Imaging and Body MRI, Beth Israel Deaconess \\ Medical Center, Harvard Medical School, Boston, Massachusetts
}

\begin{abstract}
BACKGROUND—Off-label magnetic resonance imaging (MRI) for patients with cardiac implantable electrical devices has been limited owing to concerns about safety and unclear diagnostic and prognostic utility.
\end{abstract}

OBJECTIVE-The purpose of this study was to define major and minor adverse events with offlabel MRI scans.

METHODS-We prospectively evaluated patients with non-MRI-conditional cardiac implantable electrical devices referred for MRI scans under a strict clinical protocol. The primary safety outcome was incidence of major adverse events (loss of pacing, inappropriate shock or antitachycardia pacing, need for system revision, or death) or minor adverse events (inappropriate pacing, arrhythmias, power-on-reset events, heating at the generator site, or changes in device parameters at baseline or at 6 months).

RESULTS-A total of 189 MRI scans were performed in 123 patients (63.1\% [78] men; median age $70 \pm 18.5$ years; $37.0 \%$ [70] patients with implantable cardioverter-defibrillators; $21.8 \%$ [41] pacemaker-dependent patients) predominantly for brain or spinal conditions. A minority of scans (22.7\% [43]) were performed for urgent or emergent indications. Major adverse events were rare: 1 patient with loss of pacing, no deaths, or system revisions (overall rate $0.5 \%$; $95 \%$ confidence interval $0.01-2.91$ ). Minor adverse events were similarly rare (overall rate $1.6 \% ; 95 \%$ confidence interval 0.3-4.6). Nearly all studies (98.4\% [186]) were interpretable, while 74.9\% [142] were determined to change management according to the prespecified criteria. No clinically significant changes were observed in device parameters acutely after MRI or at 6 months as compared with baseline across all patient and device categories.

Address reprint requests and correspondence: Dr Daniel B. Kramer, Richard A. and Susan F. Smith Center for Cardiovascular Outcomes Research, Division of Cardiovascular Medicine, Beth Israel Deaconess Medical Center, 375 Longwood Avenue, Fourth Floor, Boston, MA 02215.dkramer@bidmc.harvard.edu. 
CONCLUSION-Off-label MRI scans performed under a strict protocol demonstrated excellent short- and medium-term safety while providing interpretable imaging that frequently influenced clinical care.

\section{Keywords}

magnetic resonance imaging; Cardiac pacemakers; Implantable cardioverter-defibrillators

\section{Introduction}

Access to magnetic resonance imaging (MRI) remains limited for most patients with cardiac implantable electrical devices (CIEDs) including pacemakers (PMs) and implantable cardioverter-defibrillators (ICDs). ${ }^{1,2}$ While a limited number of CIEDs are now Food and Drug Administration approved for safe use in the MRI environment, the majority of devices currently in clinical use lack this labeling. Safety concerns surrounding off-label MRI scans include device migration, system failure, and lead tip heating resulting in acute arrhythmias or long-term changes in lead parameters. ${ }^{1}$ The current American Heart Association guidelines recommend against the use of MRI in patients with non-MRI-conditional CIEDs, particularly PM-dependent patients and those with ICDs. ${ }^{3}$ In addition, device manufacturers and the Food and Drug Administration do not support "off-label" MRI for patients with non-MRI-conditional CIEDs. ${ }^{4-7}$

Although studies have demonstrated the safety of MRI in well-selected patients with CIEDs, ${ }^{8-11}$ replicating these outcomes at additional centers remains an important step toward broader clinical application. There are also fewer data on PM-dependent patients with ICDs, who represent a higher-risk subset of patients with CIEDs who were excluded from prior studies ${ }^{11}$ as well as the MagnaSafe Registry. ${ }^{12}$ There are also limited data on MRI scanning of the thoracic region and the consequences of direct radiofrequency irradiation of the CIED. ${ }^{12}$ Lastly, the impact of MRI scans on the clinical care of patients with CIEDs has not been rigorously established. Thus, the goals of this study were to prospectively evaluate the safety and clinical utility of MRI scans in patients with non-MRIconditional devices.

\section{Methods}

\section{Study design and setting}

This study was performed at Beth Israel Deaconess Medical Center, an academic referral center in Boston, MA. A clinical protocol for evaluating patients with CIEDs referred for MRI scans was developed in collaboration with radiology and cardiac electrophysiology on the basis of a previously published protocol with a known safety record for MRI scans on off-label CIEDs, ${ }^{13}$ with data prospectively collected using standardized case report forms embedded in the electronic medical record as well as the Research Electronic Data Capture (REDCap) system, a secure Web-based data capture application. ${ }^{14}$ This study was approved by the institutional review board for the Beth Israel Deaconess Medical Center. 


\section{Study population}

Patients older than 18 years with CIEDs referred to the Beth Israel Deaconess Medical

Center for MRI were prospectively enrolled between June 19, 2014, and October 19, 2016.

Patients whose CIEDs were implanted for $<6$ weeks were excluded from the clinical protocol, consistent with prior published protocols. ${ }^{13}$ The presence of capped or abandoned leads or nontransvenous epicardial leads was considered relative contraindications out of concern for higher risk of lead heating, with exceptions made only on a case-by-case basis. ${ }^{13}$ Devices implanted before the year 2000 were excluded. ${ }^{13}$ There were no restrictions based on PM dependence, regardless of the CIED system.

\section{Clinical protocol}

Figure 1 summarizes the clinical protocol for performing MRI scans in patients with CIEDs. ${ }^{13}$ All cases referred for MRI scans were first reviewed by a cardiac electrophysiology attending. Case review included assessment of the patient's current CIED system and implant indications, including adjudication of current battery and lead parameters and PM dependence. The clinical circumstances and indications for the requested MRI were also reviewed, and when necessary, clarifying questions were sent by email to the referring providers. In concert with this assessment, a radiology attending from the relevant target area (such as neuroradiology, abdominal radiology, or musculoskeletal radiology) reviewed each request for the suitability of MRI to answer the relevant clinical question. Both attendings documented approval of MRI before scheduling.

At the time of MRI, a physician from the clinical electrophysiology service performed a full device interrogation before the scan to confirm current battery and lead parameters and to save pre-MRI programmed settings. For patients with ICDs, all tachycardia detection and therapy was turned off. For both ICDs and PMs, PM-dependent patients were placed in an asynchronous pacing mode (AOO, VOO, or DOO according to device type) with pacing output changed to $5 \mathrm{~V}$ amplitude. Nondependent patients were placed in demand mode (AAI, VVI, or DDI), and any additional features (rate response and ventricular sense response) that could impact pacing therapy were disabled. While in the MRI scanner, all patients were monitored using wireless electrocardiographic telemetry as well as pulse oximetry with concurrent voice contact. After each MRI, devices were re-interrogated, battery and lead parameters checked, and original settings restored.

A 1.5-T magnet was used for all studies. The radiology attending approving the study provided final protocol information to limit the specific absorption rate to $2.0 \mathrm{~W} / \mathrm{kg}$ on the basis of prior literature. ${ }^{13}$ No specific software algorithms to minimize artifact from the CIED itself were used.

\section{Variables}

Data on patient demographic characteristics, clinical and imaging parameters, device and lead characteristics, and adverse events were ascertained at the time of imaging and at 6 months from electronic health records. 
Demographic characteristics noted included age and insurance type (Medicare, Medicaid, private insurance, or none). Clinical variables noted included the target area of the imaging (brain, cervical/thoracic/lumbar spine, abdomen, upper/lower extremity, or other), and inpatient vs outpatient status. In addition, we noted whether cases were emergent (completed within 2 hours of requisition), urgent (completed within 48 hours), or elective.

Device characteristics recorded included the device type (PM or ICD and single/dual/ biventricular system), presence of any capped/abandoned leads, and PM dependence (defined as stable escape rhythm $>30$ beats/min). The manufacturer, model number, and implant date of the generator and leads were noted. The battery life, sensing (in millivolts), impedance (in ohms), and pacing threshold (amplitude and pulse width) were recorded before and after each MRI and at 6 months.

Major adverse events included acute loss of pacing, inappropriate shock or antitachycardia pacing, need for system revision, or death. Minor adverse events included clinically significant inappropriate pacing (defined as symptoms or hemodynamic compromise resulting from undersensing or inappropriately asynchronous pacing), clinically significant arrhythmias occurring within 15 minutes of the MRI period, power-on-reset events, or heating or discomfort at the generator site. We also considered as minor adverse events changes in device parameters (either acutely or at 6 months) that did not lead to need for system revision, specifically acute variation in pre-/post-MRI capture thresholds $250 \%$, acute variation in pre-/post-MRI lead impedance $230 \%$, and acute variation in pre-/postMRI P-/R-wave amplitude $250 \%$.

Lastly, utility of the imaging study was assessed via medical record review by 2 independent cardiologists (J.B.S. and J.B.W.) using a standardized 4-item scale. Items included were as follows: (1) Was the study interpretable? (2) Did MRI provide a new diagnosis? (3) Did MRI confirm a prior diagnosis? and (4) Was there a change in treatment plan, a subsequent intervention, or a change in prognosis attributable to MRI? For analytical purposes, a study was adjudicated as useful if the study was deemed to be interpretable and had at least 1 affirmative response to the other 3 items on the utility scale.

\section{Statistical analysis}

For variables describing the study population, categorical data were expressed as frequencies and percentages and continuous data as means and SDs.

For the evaluation of MRI safety, the incidence rates of major and minor adverse events were determined at the time of imaging, with $95 \%$ confidence intervals (CIs) determined using the exact method based on binomial distributions.

To evaluate immediate and long-term parameter changes, comparisons of mean differences were made between pre-and post-MRI parameters, and between pre-MRI and 6-month parameters. To account for correlation of repeated outcomes within a given individual, linear mixed-effects models were used to determine the mean difference, $95 \% \mathrm{CI}$, and $P$ value for comparisons of device parameter changes over time, where subject was treated as random effect and study was treated as random effect nested within subject (except that battery value 
was compared with subject as the only random effect due to limited number of studies with available data). To account for missing 6-month follow-up values (driven by patients with longitudinal care outside of Beth Israel Deaconess Medical Center), missingness was treated as noninformative and comparisons were made of the available data.

Lastly, the proportion of studies adjudicated as useful was calculated, with using Cohen's $~ \kappa$ statistics for interrater agreement for each item in the scale. A study was defined as useful if the study was deemed to be interpretable and had at least 1 affirmative response to the other 3 items on the utility scale. The $95 \%$ CIs for $\mathrm{\kappa}$ statistics were computed, with standard errors estimated using bootstrap resampling where the resampling unit is each subject. Thus, the standard errors were adjusted for within-individual correlations, as a given individual may have had multiple MRI scans.

All analyses were conducted with SAS version 9.4 (SAS Institute Inc., Cary, NC). $P$ values were 2 sided, and an a level of .05 was considered statistically significant.

\section{Results}

\section{Cohort characteristics}

A total of 123 patients (median age $70 \pm 18.5$ years; $61.9 \%$ Medicare beneficiaries) were enrolled over 29 months, undergoing 189 separate MRI scans. Most MRI scans (77.2\%) were performed as outpatient studies, and accordingly $77 \%$ were considered elective while $20.6 \%$ were deemed urgent and $2.1 \%$ emergent. The majority of patients (63\%) had PMs, and $21.8 \%$ were considered PM dependent.

Of 189 MRI scans, 232 body sites were imaged, as $>1$ body site could be imaged in a given study. The area most frequently imaged was the brain (50.8\%) followed by cervical and lumbar spine (both $21.7 \%$ ), thoracic spine (14.8\%), abdomen/pelvis (6.9\%), lower extremity (4.2\%), upper extremity (1.6\%), and other (1.1\%) (Figure 2 ).

The most common generator brand was Medtronic (56.6\%) followed by St. Jude Medical (21.2\%), Boston Scientific or Guidant (19.0\%), Biotronik (2.6\%), and Sorin-ELA (0.5\%). Nearly all patients (96.2\%) had right ventricular leads, $77.4 \%$ had right atrial leads, and $11.3 \%$ had coronary sinus leads. Four patients $(2.2 \%)$ had subcutaneous devices, and only 2 patients $(1.1 \%)$ had additional capped leads.

\section{Major and minor adverse events}

No patients required system revision at baseline or 6 months, and there were no deaths. Adverse events were rare, with only 1 major adverse event (overall rate $0.5 \%$; 95\% CI $0.01-$ 2.91). One outpatient with complete heart block and a Medtronic Sigma SDR generator and Medtronic 4076 and 4092 right atrial and right ventricular leads experienced acute loss of pacing. The patient had been programmed D00 80 for MRI and, upon entry into the MRI room, was noted immediately to have a heart rate of 40 with a narrow QRS junctional escape. The patient was removed from zone 4 to zone 3 and the device re-interrogated, demonstrating a power-on-reset event with automatic conversion to VVI 65. After subsequent Medtronic review, absence of pacing at 65 beats/min was attributed to inhibition 
of pacing by electromagnetic interference from the static magnetic field with subsequent oversensing. The patient was asymptomatic with the junctional rhythm, and MRI was performed in DOO mode as planned, with the patient in a junctional rhythm without sequelae. Post-MRI, in zone 3 , the device continued to a report a power-on-reset alert, but device and lead parameters were rechecked and found to be stable from initial measurements. The original settings were restored without subsequent complication. As the generator had been close to the elective replacement interval before MRI, the patient was discharged home and electively readmitted 9 months later for a generator change.

There were 3 minor adverse events (overall rate $1.6 \%$; 95\% CI 0.3-4.6). One patient with a St. Jude Medical Assurity generator and St. Jude Medical 1088TC and 2088TC leads developed atrial arrhythmia during MRI, which was self-limiting and without clinical consequences. Heating or discomfort at the generator site developed during MRI in 2 patients, one with a Medtronic Adapta L generator and Medtronic 4076 atrial and ventricular leads and the other with a Boston Scientific Altrua generator with St. Jude Medical 1688TC atrial and ventricular leads. The heating and discomfort described improved upon immediate cessation of MRI.

At 6 months, 78 individuals (85.7\%) were alive and 66 (83.5\%) had device follow-up available (Table 1). There were no significant differences in battery values before and after MRI, but there was a small but significant decline in battery value as measured in years from pre-MRI to 6 months (mean difference- 0.32 years; $95 \% \mathrm{CI}-0.56$ to -0.08 years; $P=.01$ ). Right atrial sensing and right atrial impedance were not different before and after MRI or at 6 months. Right atrial threshold values were unchanged before and after MRI but increased slightly over 6 months (mean difference $0.07 \mathrm{mV} ; 95 \%$ CI $0.01-0.12 \mathrm{mV} ; P=.03$ at a mean of $0.4 \pm 0.1 \mathrm{~ms}$ ). Right ventricular lead sensing, impedance, and threshold measurements were unchanged before and after MRI and at 6 months as were coronary sinus lead measurements.

\section{Clinical utility}

Nearly all studies were considered interpretable, with moderate agreement (98.9\% agreement; $\kappa=0.66 ; 95 \%$ CI $0.22-1.00$ ), and $79.6 \%$ met our prespecified criteria for "useful" (interpretable and led to a change in treatment, subsequent procedure, or a change in prognosis). Considering each question separately, a majority of studies provided a new diagnosis with moderate agreement (77.3\% agreement; $\kappa=0.54 ; 95 \%$ CI $0.42-0.66$ ). Half of all studies were considered to have confirmed a prior diagnosis with moderate agreement (80.4\% agreement; $\kappa=0.61 ; 95 \%$ CI $0.50-0.72)$. A large majority of studies resulted in a change in treatment plan, a subsequent intervention, or a change in prognosis with moderate agreement (84.7\% agreement; $\kappa=0.53 ; 95 \%$ CI $0.38-0.68)$.

\section{Discussion}

This single-center report reinforces the safety and clinical utility of performing off-label MRI scans for patients with CIEDs, including those who are PM dependent. A strict clinical protocol yielded MRI scans that influenced clinical care, with no system revisions, deaths, serious arrhythmias, or clinically significant changes in device parameters at 6 months of 
follow-up. These results add to a growing published experience illustrating that patients with nearly any CIED can access MRI without serious clinical consequences if appropriate precautions are followed.

Our study builds on prior reports using similar clinical protocols that have demonstrated the feasibility and safety of MRI scans in this setting, ${ }^{4-7}$ particularly for patients receiving thoracic imaging. Thus, our more permissive protocol, which also included subcutaneous ICDs, thoracic scans, and 2 patients with capped leads, suggests that the generally favorable results of larger studies can be extended to higher-risk patients and other centers.

These results may also alleviate concerns about long-term consequences of MRI scans on CIED function. While there was a small decline in battery value as measured in years of battery life remaining and a small increase in right atrial lead threshold potential between the pre-MRI interrogation and 6 months, the absolute magnitude of these changes is small and unlikely to be clinically meaningful. No other significant changes in device parameters were observed. This contrasts with the recently published MagnaSafe Registry, which described a clinically relevant device parameter change in $8.8 \%$ of patients with PMs and $7.1 \%$ of patients with ICDs. ${ }^{12}$ As this registry excluded PM-dependent individuals with ICDs and MRI scans of the thorax, the present study extends safety data to include these populations. ${ }^{12}$ However, even if small changes in device parameters are noted in a minority of cases, we submit that system revision is the more relevant end point from a patient's perspective, particularly given the clinical context for most MRI scans. Nevertheless, while the small increase in right atrial thresholds observed over 6 months may be artifactual, given the theoretical possibility of transmission of radiofrequency energy to the leads during MRI, possible mild thermal injury at the lead tips causing threshold elevations cannot be ruled out and requires further study. In addition, while some studies suggest routine monitoring is not necessary, ${ }^{15}$ as 1 potentially life-threatening power-on-reset event occurred during the study, our study argues that it remains necessary to perform off-label MRI scans under a strict protocol in a clinically monitored setting with trained personnel. Moreover, while power-onreset events have been described in MRI scans of CIEDs, ${ }^{16-20}$ it is unknown whether the majority of these events are specific to a single vendor, product, or magnetic field zone.

To our knowledge, no prior study has reported the clinical utility of these closely vetted MRI scans, which required approval from both a radiologist and a cardiac electrophysiologist before scheduling. Specifically, involvement of radiology staff confirms that the area of interest will be evaluable even in the presence of expected device-related artifact and that MRI is the appropriate modality to pursue rather than computed tomography, ultrasound, or other strategies. At the same time, review of each case by a cardiac electrophysiologist provides not just review of the device-related information but also review of the clinical indications as well as opportunities to communicate directly with the referring providers to clarify whether and in what way MRI is likely to influence clinical care. Accordingly, our findings of $98.4 \%$ studies being interpretable and $79.6 \%$ meeting our prespecified criteria for "useful" (interpretable and led to a change in treatment, subsequent procedure, or a change in prognosis) support the adoption of similar protocols else-where to broaden patients' access to this important modality. 


\section{Study limitations}

Our study includes potential limitations. As a convenience sample at a large academic teaching hospital, the study cohort is subject to referral bias and the results may not generalize to other settings and clinical environments. In addition, the results may not generalize to other clinical protocols for performing MRI scans on off-label CIEDs. ${ }^{21-28} \mathrm{We}$ did not have a control group of patients who underwent MRI for comparison of utility. Similarly, for our analysis of device parameters, we did not have a control group of patients with CIEDs who did not undergo MRI. Complete follow-up at 6 months was not available for all patients, and we had only limited numbers of patients with subcutaneous ICDs and capped/abandoned leads, which limits generalizability of our findings to these small but important populations. In addition, the slight increase in atrial thresholds over time suggests that lead tip heating and thermal injury potential cannot be exonerated, especially in the context of multiple MRI studies and warrants further study. However, these risks serve to emphasize the importance of a case-by-case consideration of the risk and benefits of MRI for individual patients. This extends to cases such as retained leads or other relative contraindications, as in many cases informed patients and a well-trained clinical team may accept these risks in order to obtain essential imaging necessary to guide lifesaving care.

\section{Conclusion}

MRI scans performed off-label on CIEDs under a strict clinical protocol and with appropriate clinical monitoring can be done safely and influence patient care.

\section{Acknowledgments}

Dr Strom was supported by a Ruth L. Kirschstein National Research Service Award (award no. 5T32HL007374-37). Dr Kramer was supported by a Paul B. Beeson Career Development Awards in Aging Research program (award no. K23AG045963) and the Greenwall Faculty Scholars Program, and he is a consultant to Circulatory Systems Advisory Panel of the Food and Drug Administration.

We thank the cardiology fellows at Beth Israel Deaconess Medical Center for providing the patient supervision necessary to support the magnetic resonance imaging protocol.

\section{References}

1. Verma A, Ha AC, Dennie C, et al. Canadian Heart Rhythm Society and Canadian Association of Radiologists consensus statement on magnetic resonance imaging with cardiac implantable electronic devices. Can J Cardiol. 2014; 30:1131-1141. [PubMed: 25262858]

2. Nazarian S, Reynolds MR, Ryan MP, Wolff SD, Mollenkopf SA, Turakhia MP. Utilization and likelihood of radiologic diagnostic imaging in patients with implantable cardiac defibrillators. J Magn Reson Imaging. 2016; 43:115-127. [PubMed: 26118943]

3. Levine GN, Gomes AS, Arai AE, Bluemke DA, Flamm SD, Kanal E, Manning WJ, Martin ET, Smith JM, Wilke N, Shellock FS. American Heart Association Committee on Diagnostic and Interventional Cardiac Catheterization; American Heart Association Council on Clinical Cardiology; American Heart Association Council on Cardiovascular Radiology and Intervention. Safety of magnetic resonance imaging in patients with cardiovascular devices: an American Heart Association scientific statement from the Committee on Diagnostic and Interventional Cardiac Catheterization, Council on Clinical Cardiology, and the Council on Cardiovascular Radiology and Intervention: endorsed by the American College of Cardiology Foundation, the North American Society for Cardiac Imaging, and the Society for Cardiovascular Magnetic Resonance. Circulation. 2007; 116:2878-2891. [PubMed: 18025533] 
4. Levine PA. Industry viewpoint: St. Jude Medical: pacemakers, ICDs and MRI. Pacing Clin Electrophysiol. 2005; 28:266-267. [PubMed: 15826255]

5. Smith JM. Industry viewpoint: Guidant: pacemakers, ICDs and MRI. Pacing Clin Electrophysiol. 2005; 28:264. [PubMed: 15826253]

6. Stanton MS. Industry viewpoint: Medtronic: pacemakers, ICDs and MRI. Pacing Clin Electrophysiol. 2005; 28:265. [PubMed: 15826254]

7. Faris OP, Shein M. Food and Drug Administration perspective: magnetic resonance imaging of pacemaker and implantable cardioverter-defibrillator patients. Circulation. 2006; 114:1232-1233. [PubMed: 16982951]

8. Boilson BA, Wokhlu A, Acker NG, Felmlee JP, Watson RE Jr, Julsrud PR, Friedman PA, Cha YM, Rea RF, Hayes DL, Shen WK. Safety of magnetic resonance imaging in patients with permanent pacemakers: a collaborative clinical approach. J Interv Card Electrophysiol. 2012; 33:59-67. [PubMed: 21938517]

9. Nordbeck P, Ertl G, Ritter O. Magnetic resonance imaging safety in pacemaker and implantable cardioverter defibrillator patients: how far have we come? Eur Heart J. 2015; 36:1505-1511. [PubMed: 25796053]

10. Sommer T, Naehle CP, Yang A, Zeijlemaker V, Hackenbroch M, Schmiedel A, Meyer C, Strach K, Skowasch D, Vahlhaus C, Litt H, Schild H. Strategy for safe performance of extrathoracic magnetic resonance imaging at 1.5 tesla in the presence of cardiac pacemakers in non-pacemakerdependent patients: a prospective study with 115 examinations. Circulation. 2006; 114:1285-1292. [PubMed: 16966587]

11. Nazarian S, Hansford R, Roguin A, et al. A prospective evaluation of a protocol for magnetic resonance imaging of patients with implanted cardiac devices. Ann Intern Med. 2011; 155:415424. [PubMed: 21969340]

12. Russo RJ, Costa HS, Silva PD, et al. Assessing the risks associated with MRI in patients with a pacemaker or defibrillator. N Engl J Med. 2017; 376:755-764. [PubMed: 28225684]

13. Nazarian S, Roguin A, Zviman MM, Lardo AC, Dickfeld TL, Calkins H, Weiss RG, Berger RD, Bluemke DA, Halperin HR. Clinical utility and safety of a protocol for noncardiac and cardiac magnetic resonance imaging of patients with permanent pacemakers and implantable-cardioverter defibrillators at 1.5 tesla. Circulation. 2006; 114:1277-1284. [PubMed: 16966586]

14. Harris PA, Taylor R, Thielke R, Payne J, Gonzalez N, Conde JG. Research Electronic Data Capture (REDCap) - a metadata-driven methodology and workflow process for providing translational research informatics support. J Biomed Inform. 2009; 42:377-381. [PubMed: 18929686]

15. Bertelsen L, Petersen HH, Philbert BT, Svendsen JH, Thomsen C, Vejlstrup N. Safety of magnetic resonance scanning without monitoring of patients with pacemakers [published online ahead of print October 12, 2016]. Europace. 2016 Oct 12. pii: euw066.

16. Jilek C, Tzeis S, Reents T, Estner HL, Fichtner S, Ammar S, Wu J, Hessling G, Deisenhofer I, Kolb C. Safety of implantable pacemakers and cardioverter defibrillators in the magnetic field of a novel remote magnetic navigation system. J Cardiovasc Electrophysiol. 2010; 21:1136-1141. [PubMed: 20522155]

17. Okamura, H., Padmanabhan, D., Watson, RE., Jr, Dalzell, C., Acker, N., Jondal, M., Romme, AL., Cha, YM., Asirvatham, SJ., Felmlee, JP., Friedman, PA. Magnetic resonance imaging in nondependent pacemaker patients with pacemakers and defibrillators with a nearly depleted battery [published online head of print March 23, 2017]. Pacing Clin Electrophysiol. 2017 Feb 7. http:// dx.doi.org/10.1111/pace.13042

18. Muehling OM, Wakili R, Greif M, von Ziegler F, Morhard D, Brueckmann H, Becker A. Immediate and 12 months follow up of function and lead integrity after cranial MRI in 356 patients with conventional cardiac pacemakers. J Cardiovasc Magn Reson. 2014; 16:39. [PubMed: 24903354]

19. Higgins JV, Sheldon SH, Watson RE Jr, Dalzell C, Acker N, Cha YM, Asirvatham SJ, Kapa S, Felmlee JP, Friedman PA. "Power-on resets" in cardiac implantable electronic devices during magnetic resonance imaging. Heart Rhythm. 2015; 12:540-544. [PubMed: 25460173]

20. Gimbel JR. Unexpected asystole during 3T magnetic resonance imaging of a pacemaker-dependent patient with a 'modern' pacemaker. Europace. 2009; 11:1241-1242. [PubMed: 19556252] 
21. Martin ET, Coman JA, Shellock FG, Pulling CC, Fair R, Jenkins K. Magnetic resonance imaging and cardiac pacemaker safety at 1.5-tesla. J Am Coll Cardiol. 2004; 43:1315-1324. [PubMed: 15063447]

22. Gimbel JR, Bailey SM, Tchou PJ, Ruggieri PM, Wilkoff BL. Strategies for the safe magnetic resonance imaging of pacemaker-dependent patients. Pacing Clin Electrophysiol. 2005; 28:10411046. [PubMed: 16221260]

23. Gimbel JR. Magnetic resonance imaging of implantable cardiac rhythm devices at 3.0 tesla. Pacing Clin Electrophysiol. 2008; 31:795-801. [PubMed: 18684275]

24. Langman DA, Finn JP, Ennis DB. Abandoned pacemaker leads are a potential risk for patients undergoing MRI. Pacing Clin Electrophysiol. 2011; 34:1051-1053. [PubMed: 21797902]

25. Langman DA, Goldberg IB, Finn JP, Ennis DB. Pacemaker lead tip heating in abandoned and pacemaker-attached leads at 1.5 tesla MRI. J Magn Reson Imaging. 2011; 33:426-431. [PubMed: 21274985]

26. Friedman HL, Acker N, Dalzell C, Shen WK, Asirvatham SJ, Cha YM, Hodge D, Felmlee J, Watson R, Friedman PA. Magnetic resonance imaging in patients with recently implanted pacemakers. Pacing Clin Electrophysiol. 2013; 36:1090-1095. [PubMed: 23826621]

27. Higgins JV, Gard JJ, Sheldon SH, Espinosa RE, Wood CP, Felmlee JP, Cha YM, Asirvatham SJ, Dalzell C, Acker N, Watson RE Jr, Friedman PA. Safety and outcomes of magnetic resonance imaging in patients with abandoned pacemaker and defibrillator leads. Pacing Clin Electrophysiol. 2014; 37:1284-1290. [PubMed: 24809591]

28. Miller JD, Nazarian S, Halperin HR. Implantable electronic cardiac devices and compatibility with magnetic resonance imaging. J Am Coll Cardiol. 2016; 68:1590-1598. [PubMed: 27687201] 


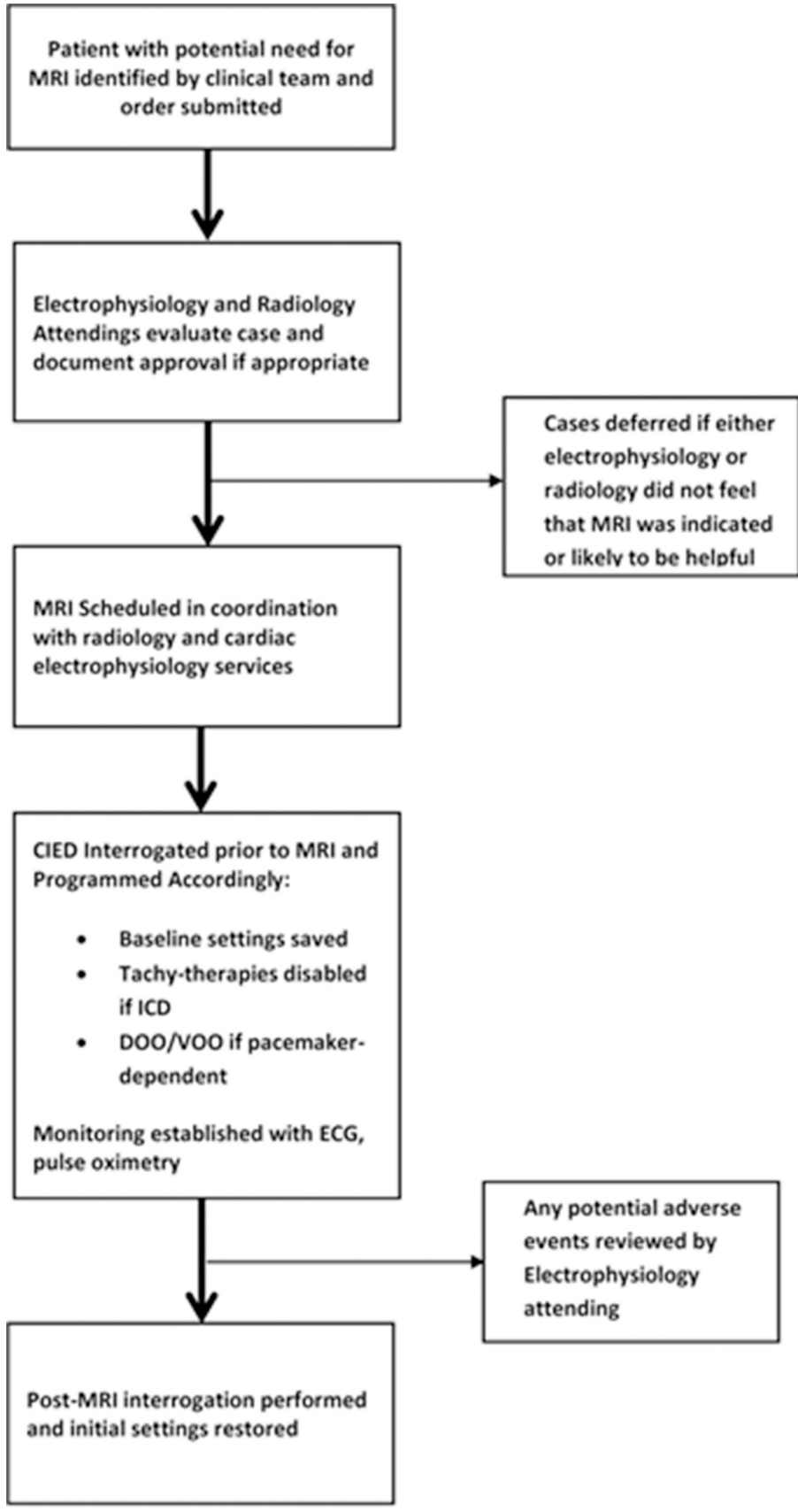

Figure 1.

Clinical protocol for performing magnetic resonance imaging (MRI) scans in patients with cardiac implantable electrical devices (CIEDs) not approved as MRI conditional. ECG = electrocardiography; ICD = implantable cardioverter-defibrillator. 


\section{Upper Extremity_ Lower Extremity}

$1 \%$

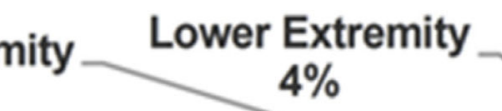

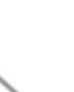

Other

Abdomen/Pelvis $5 \%$

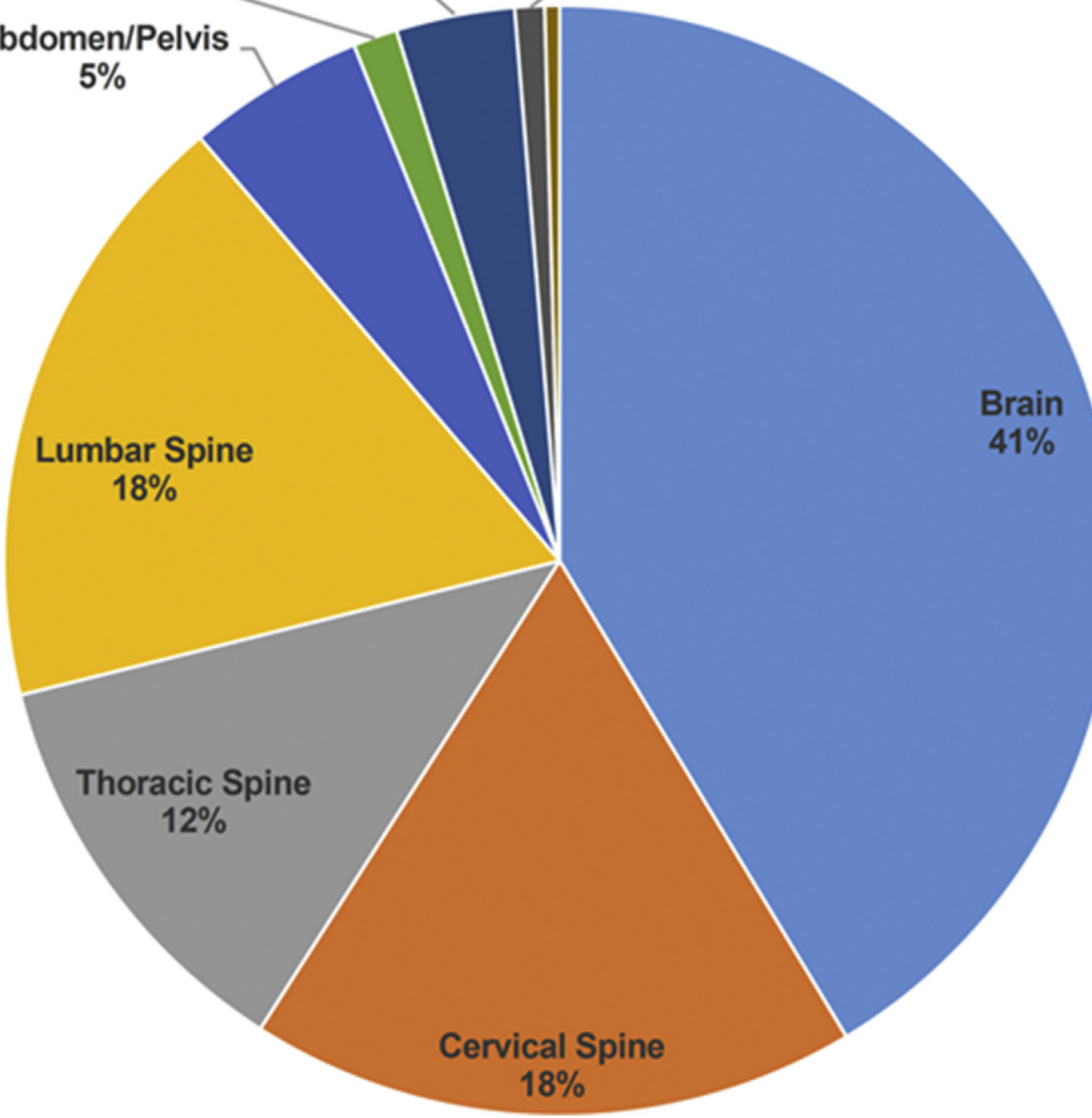

Figure 2.

Proportion of magnetic resonance imaging scans $(n=5232)$ performed according to body site. 


\section{롤 \\ ㄱ.}

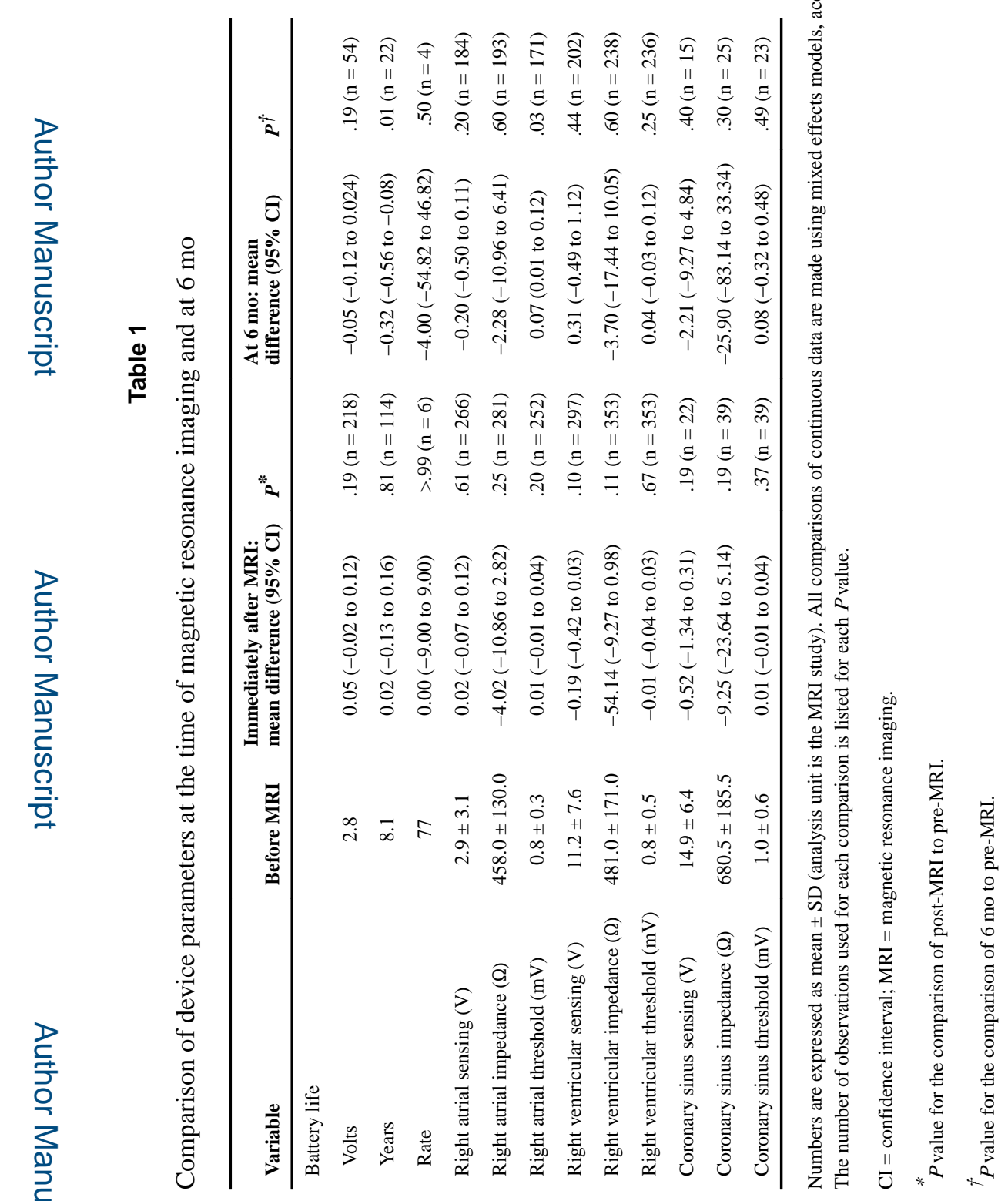

Heart Rhythm. Author manuscript; available in PMC 2017 August 15. 